\title{
OPTIMIZATION OF CURRENT EXCITATION FOR PERMANENT MAGNET LINEAR SYNCHRONOUS MOTORS
}

\author{
Christof Röhrig \\ University of Applied Sciences Dortmund \\ Emil-Figge-Str. 42, 44227 Dortmund, Germany \\ Email: christof.roehrig@fh-dortmund.de
}

Keywords: Optimization, motor control, force ripple, linear synchronous motors

Abstract: The main problem in improving the tracking performance of permanent magnet linear synchronous motors is the presence of force ripple caused by mismatched current excitation. This paper presents a method to optimize the current excitation of the motors in order to generate smooth force. The optimized phase current waveforms produce minimal ohmic losses and maximize motor efficiency. The current waveforms are valid for any velocity and any desired thrust force. The proposed optimization method consist of three stages. In every stage different harmonic waves of the force ripple are reduced. A comparison of the tracking performance with optimized waveforms and with sinusoidal waveforms shows the effectiveness of the method.

\section{INTRODUCTION}

Permanent magnet (PM) linear synchronous motors (LSM) are beginning to find widespread industrial applications, particularly for tasks requiring a high precision in positioning such as various semiconductor fabrication and inspection processes (Basak, 1996). PM LSMs have better performance and higher power density than their induction counterparts (Gieras and Zbigniew, 1999). The main benefits of PM LSMs are the high force density achievable and the high positioning precision and accuracy associated with the mechanical simplicity of such systems. The electromagnetic force is applied directly to the payload without any mechanical transmission such as chains or screw couplings. Todays state-ofthe-art linear motors can, typically, achieve velocities up to $10 \mathrm{~m} / \mathrm{s}$ and accelerations of $25 \mathrm{~g}$ (Cassat et al., 2003).

The more predominant nonlinear effects underlying a PM LSM system are friction and force ripple arising from imperfections in the underlying components. In order to avoid force ripple different methods have been developed. In(Jahns and Soong, 1996) several techniques of torque ripple minimization for rotating motors are reviewed. In (Van den Braembussche et al., 1996) a force ripple model is developed and identification is carried out with a force sensor and a frictionless air bearing support of the motor carriage. In (Otten et al., 1997) a neuronal-network based feedforward controller is proposed to reduce the effect of force ripple. Position-triggered repetitive control is presented in (Van den Braembussche et al., 1998). Other approaches are based on disturbance observers (Schrijver and van Dijk, 1999), (Lin et al., 2000), iterative learning control (Lee et al., 2000) or adaptive control (Xu and Yao, 2000). The main problem in adaptive control is the low signal-to-noise ratio at high motor speeds (Seguritan and Rotunno, 2002). In (Röhrig and Jochheim, 2001) a force ripple compensation method for PM LSM systems with electronic commutated servo amplifiers was presented. A model based method was chosen, because force ripple is a highly reproducible and time-invariant disturbance. The parameters of the force ripple are identified in an offline procedure.

In this paper a force ripple compensation method for software commutated servo amplifiers is proposed. The software commutation requires two current command signals, from the motion controller. This two input signals of the amplifier are used to optimize the operation of the motor. The paper proposes a method for the design of the current command signals for minimization of force ripple an maximization of motor efficiency. The waveforms of the phase currents are optimized in order to get smooth force and minimal copper losses. In order to optimize the currents waveforms the force functions of the phases are 
identified. The identification is performed by measuring the force command signal in a closed position control loop. The waveform generation is directly integrated in the software commutation module of the motion controller. The optimal current waveforms are approximated with Fourier series.

The paper is organized as follows: In Section 2 the experimental setup is described. In Section 3, a physical model of the PM LSM is derived and explained. In Section 4 the optimization of current excitation is described. In Section 5 the controller design is presented and a comparison of the tracking performance with and without optimization of the current excitation is given. Finally Section 6 concludes the paper.

\section{EXPERIMENTAL SETUP}

\subsection{Linear Motor}

The motors considered here are PM LSM with epoxy cores. A PM LSM consists of a secondary and a moving primary. There are two basic classifications of PM LSMs: epoxy core (i.e. non-ferrous, slotless) and iron core. Epoxy core motors have coils wound within epoxy support. These motors have a closed magnetic path through the gap since two magnetic plates "sandwich" the coil assembly (Anorad, 1999). Figure 1 shows an unmounted PM LSM with epoxy core. The secondary induces a multipole magnetic field in

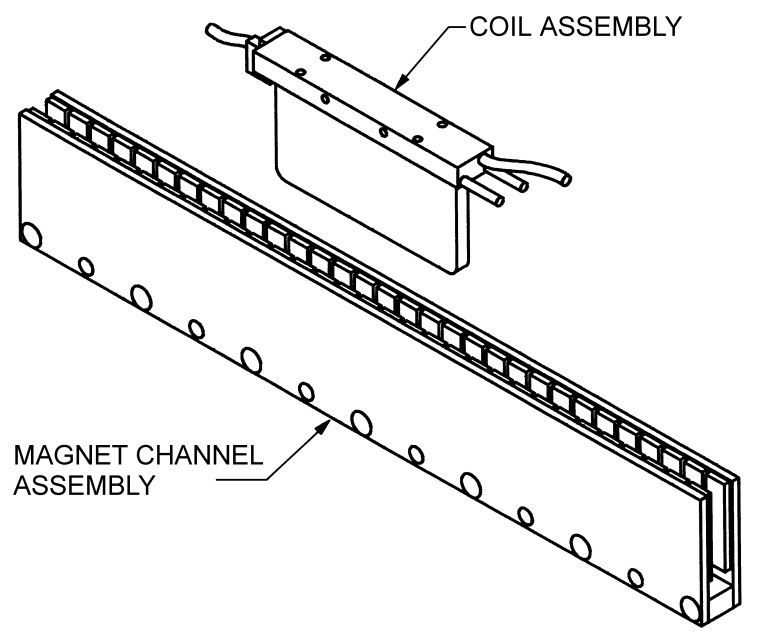

Figure 1: Anorad LE linear motor

the air gap between the magnetic plates. The magnet assembly consists of rare earth magnets, mounted in alternate polarity on the steel plates. The electromagnetic thrust force is produced by the interac- tion between the permanent magnetic field in the secondary and the magnetic field in the primary driven by the phase currents of the servo amplifier. The linear motor under evaluation is a current-controlled threephase motor driving a carriage supported by roller bearings. The motor drives a mass of total $1.5 \mathrm{Kg}$ and is vertically mounted.

\subsection{Servo Amplifier}

The servo amplifiers employed in the setup are PWM types with closed current control loop. The software commutation of the three phases is performed in the motion controller with the help of the position encoder. This commutation method requires two current command signals, from the controller. The initialization routine for determining the phase relationship is part of the motion controller. The third phase current depends on the others, because of the star connection.

$$
i_{C}=-i_{A}-i_{B}
$$

The maximum input signals $u_{A}, u_{B}$ of the servo amplifier $( \pm 10 \mathrm{~V})$ correlate to the peak currents of the current loops. In the setup the peak current of the amplifier is $25 A$. The PWM works with a switching frequency of $24 k \mathrm{~Hz}$. The current loop bandwidth is specified with 2.5kHz. (Anorad, 1998)

\section{SYSTEM MODELING}

The thrust force is produced by interaction between the permanent magnetic field in the secondary and the electromagnetic field of the phase windings. The thrust force is proportional to the magnetic field and the phase currents $i_{A}, i_{B}, i_{C}$. The back-EMF (electromotive force) induced in a phase winding $\left(e_{A}, e_{B}\right.$, $\left.e_{C}\right)$ is proportional to the magnetic field and the speed of the motor. The total thrust force $F_{\text {thrust }}$ is the sum of the forces produced by each phases:

$$
\dot{x} F_{\text {thrust }}=\sum_{p} e_{p}(x) i_{p} ; \quad p \in\{A, B, C\}
$$

The back-EMF waveforms $\frac{e_{p}}{\dot{x}}$ can also be interpreted as the force functions of the phases $\left(K_{M_{p}}(x)\right)$.

$$
F_{\text {thrust }}=\sum_{p} K_{M_{p}}(x) i_{p} ; \quad p \in\{A, B, C\}
$$

There are two types of position dependent disturbances: cogging force and force ripple. Cogging is a magnetic disturbance force that is caused by attraction between the PMs and the iron part of the primary. The force depends on the relative position of the primary 
with respect to the magnets, and it is independent of the motor current. Cogging is negligible in motors with iron-less primaries (Anorad, 1999).

Force ripple is an electro-magnetic effect and causes a periodic variation of the force constant. There are two physical phenomena which lead to force ripple: Reluctance force and harmonics in the electromagnetic force. Reluctance force occurs only in motors with interior mounted PMs. In this type of motor the reluctance of the motor is a function of the position. The self inductance of the phase windings varies with the position of the primary with respect to the secondary. When current flows, this causes a position dependent force. If the PMs are surface mounted, the reluctance is constant and reluctance force is negligible.

In ironless and slotless motors the only source of force ripple are harmonics in the electromagnetic force. Only if the back-EMF waveforms are sinusoidal and balanced, symmetric sinusoidal commutation of the phase currents produces smooth force. Force ripple occurs if the motor current is different from zero, and its absolute value depends on the required thrust force and the relative position of the primary with respect to the secondary.

There are several sources of force ripple:

- motor

- harmonics of back-EMFs

- amplitude imbalance of back-EMFs

- phase imbalance of back-EMFs

- amplifier

- offset currents

- imbalance of current gains

Offset currents lead to force ripple with the same period as the commutation period. This force ripple is independent of the desired thrust force. Amplitude or phase imbalance of the motor and imbalance of amplifier gains lead to force ripple with half commutation period which scale in direct proportion to the desired thrust force. A $k^{t h}$ order harmonic of a backEMF produces $(k-1)^{t h}$ and $(k+1)^{t h}$ order harmonic force ripple if sinusoidal currents are applied.

Force ripple with the same period as the commutation period is independent of the desired current, all higher order harmonics scale in direct proportion to the desired current because of the linearity of the force equation (2).

\section{OPTIMIZATION OF CURRENT EXCITATION}

The method for optimization of current excitation consist of three stages. In every stage different har- monics of the force ripple spectrum are reduced. In order to optimize the waveforms of the currents, identification of the force functions $K_{M_{p}}(x)$ is essential. The main idea of the proposed method is to identify the force functions in a closed position control loop by measuring the force command signal $u$ of the position controller at constant load force $F_{\text {load }}$ as a function of the position $x$. Neither additional sensor nor device for position adjustment are necessary. In the experimental setup the constant load force is produced by the force of gravitation. In order to avoid inaccuracy by stiction the measurement is achieved with moving carriage. The position of the carriage is obtained from an incremental linear optical encoder with a measurement resolution of $0.1 \mu \mathrm{m}$.

\subsection{Experimental Analysis of Force Ripple}

In order to analyze the force ripple of the motor a sinusoidal reference current is applied:

$$
\begin{aligned}
u_{A}(u, \vartheta) & =u \frac{2}{3} \sin (\vartheta(x))+o_{A} \\
u_{B}(u, \vartheta) & =u \frac{2}{3} \sin \left(\vartheta(x)+\frac{2 \pi}{3}\right)+o_{B} \\
\text { with } \vartheta(x) & =\frac{\pi}{\tau_{p}}\left(x-x_{0}\right)
\end{aligned}
$$

where $u_{A}, u_{B}$ are the current commands of the two phases, $u$ is the output of the position controller (force command), $x$ is the position of the carriage, $\tau_{p}$ is the pole pitch and $x_{0}$ is the zero position with maximum force. In the first stage, the DC components of the command signals $\left(o_{A}, o_{B}\right)$ are chosen equal zero. Figure 2 shows the control signals $u, u_{A}, u_{B}$ versus the position $x$. The ripple on the force command signal $u$ is caused by force ripple. The controller compensates the force ripple by changing the force command signal over the position. If the speed of the motor is high, the force ripple increases the tracking error.

Frequency domain analyses of the force command indicates that the fundamental corresponds to the commutation period $2 \tau_{p}$. In order to estimate the parameters of the ripple a least square estimation of the model parameters (5) was applied. A least square estimation is chosen, because noise overlays the force command signal.

$$
\begin{array}{r}
f(x, \boldsymbol{\theta})=\theta_{1}+\theta_{2} x+\sum_{k=1}^{N}\left(\theta_{2 k+1} \sin \left(k \pi \frac{x}{\tau_{p}}\right)\right. \\
\left.+\theta_{2 k+2} \cos \left(k \pi \frac{x}{\tau_{p}}\right)\right)
\end{array}
$$




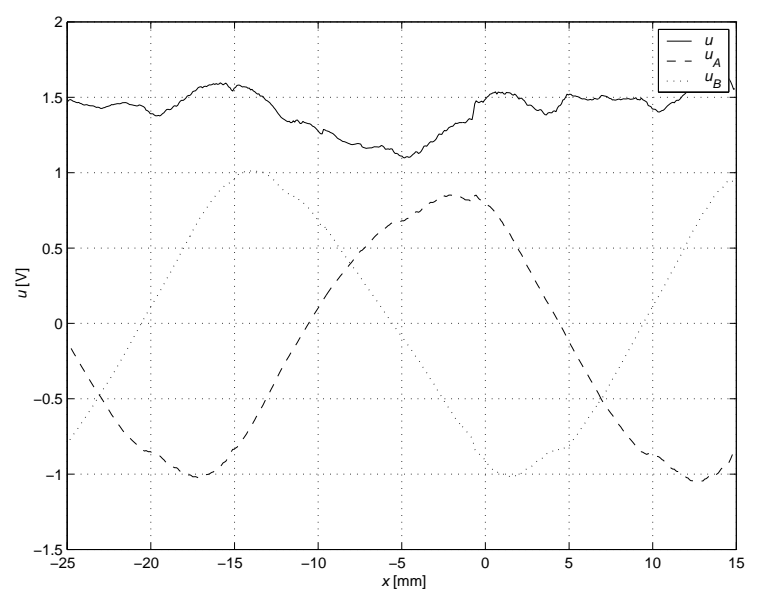

Figure 2: Phase currents at load force $F_{\text {load }}=20 \mathrm{~N}$

where $\theta_{k}$ are the estimated parameters. With $\theta_{1}$ the sum of load force and friction is estimated. The modeled spring force $\left(\theta_{2}\right)$ is necessary, because the force command signal rises with rising positions. This is caused by wire chains. Figure 3 shows the amplitudes of the sinusoids $\sqrt{\theta_{2 k+1}^{2}+\theta_{2 k+2}^{2}}$ versus the order of the harmonics $k$.

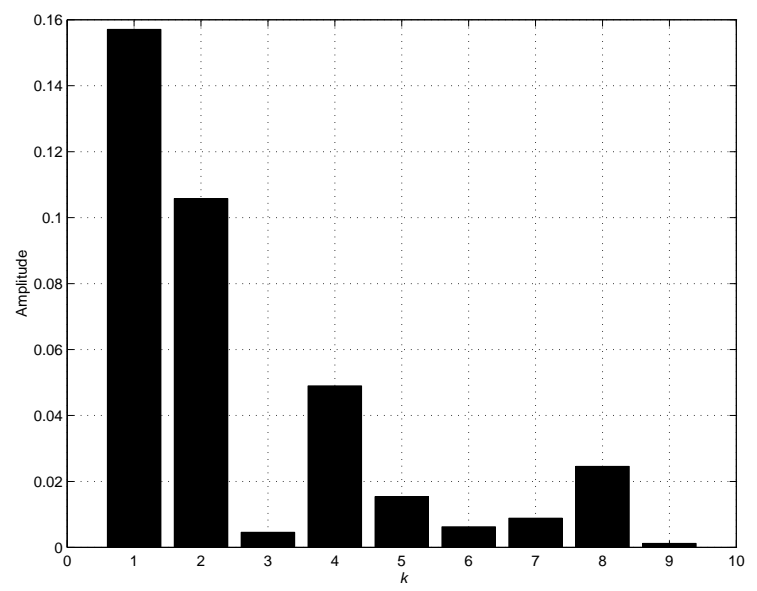

Figure 3: Spectrum of the force command

The fundamental period $(k=1)$ corresponds to $2 \tau_{p}(30 \mathrm{~mm})$. The amplitude of this sinusoid is independent of the load force. The higher order harmonics $(k>1)$ scale in direct proportion to the load force because of the linearity of the force equation 3 .

\subsection{Compensation of Command Independent Force Ripple}

The curve of the phase current command $u_{A}$ in figure 2 shows that this command independent ripple produces a DC component of the current command. This DC component compensates offsets in the analog circuits of the servo amplifier. In the first stage the DC components of the phase current commands are calculated and applied in (4) as

$$
\begin{aligned}
o_{A} & =\frac{a}{\sqrt{3}} \cos \left(\alpha-\frac{\pi}{6}\right)-\frac{a}{3} \sin \left(\alpha-\frac{\pi}{6}\right) \\
o_{B} & =\frac{2 a}{3} \sin \left(\alpha-\frac{\pi}{6}\right)
\end{aligned}
$$

where

$$
a=\sqrt{\theta_{3}^{2}+\theta_{4}^{2}} \text { and } \alpha=\arctan \frac{\theta_{4}}{\theta_{3}} .
$$

In an servo system with iron-less motor the DC components compensate the offsets of the amplifier. If an iron motor is employed, the DC components generate a sinusoidal force which compensates the cogging force.

\subsection{Identification of Force Functions}

In the next stage the force functions of the phases are identified. In order to optimize the current waveforms, it is essential to identify the amplitudes and phases of the force functions properly. The motor efficiency depends directly on a properly identified commutation zero position $x_{0}$ which depends on the phase lag of the force functions. In (Röhrig and Jochheim, 2002) a sinusoidal commutation is applied to identify the force functions. By means of sinusoidal commutation it is impossible to identify the force functions idenpendently. In this paper a square-wave commutation is applied, in order to identify the force functions independently:

$$
u_{A}(u, \vartheta)=\left\{\begin{aligned}
0 & : & 0 \leq \vartheta & <\frac{\pi}{6} \\
\frac{u}{\sqrt{3}} & : & \frac{\pi}{6} \leq \vartheta & <\frac{5 \pi}{6} \\
0 & : & \frac{5 \pi}{6} \leq \vartheta & <\frac{7 \pi}{6} \\
-\frac{u}{\sqrt{3}} & : & \frac{7 \pi}{6} \leq \vartheta & <\frac{11 \pi}{6} \\
0 & : & \frac{11 \pi}{6} \leq \vartheta & <2 \pi
\end{aligned}\right.
$$

The second phase command signal is $\frac{2 \pi}{3}$ apart:

$$
u_{B}(u, \vartheta)=u_{A}\left(u, \vartheta+\frac{2 \pi}{3}\right)
$$




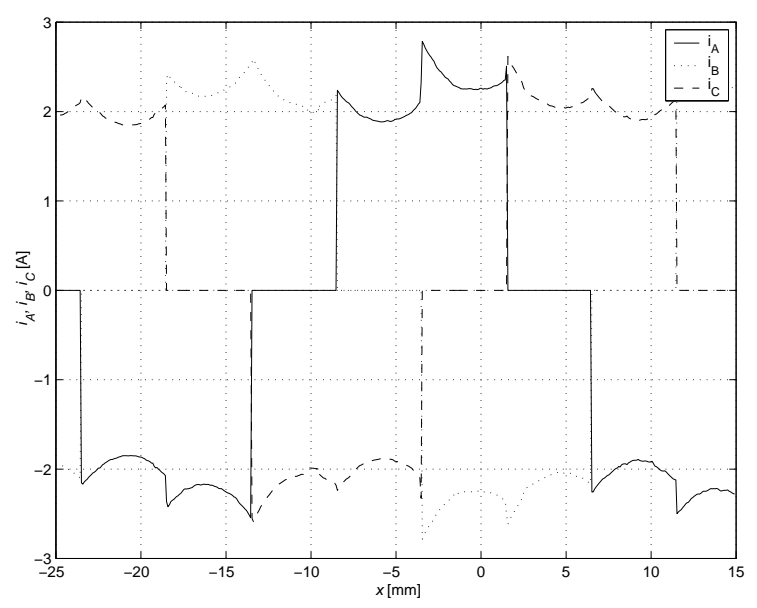

Figure 4: square-wave commutation

Figure 4 shows the phase currents $i_{A}, i_{B}, i_{C}$ versus the position $x$, when square-wave commutation is applied. The minimum values of the phase currents correlate to the maximum values of the force functions. In square-wave commutation always two phases share the same absolute value of current, which depends on the force command $u$ and the current gains of the amplifier $K_{S_{p}}$.

$$
\left|i_{p}\right|=K_{S_{p}} \frac{u}{\sqrt{3}} ; \quad p \in\{A, B\}
$$

Since only two phase currents are independent, the force equation can be described as:

$$
F_{\text {thrust }}=K_{A}(\vartheta) u_{A}+K_{B}(\vartheta) u_{B}
$$

where $K_{A}(\vartheta)$ and $K_{B}(\vartheta)$ are the force functions of the current commands $u_{A}$ and $u_{B}$ respectively.

$$
\frac{\sqrt{3} F_{\text {thrust }}}{u(\vartheta)}=\left\{\begin{array}{rlrr}
K_{B}(\vartheta) & : & -\frac{\pi}{6} \leq \vartheta & <\frac{\pi}{6} \\
K_{A}(\vartheta) & : & \frac{\pi}{6} \leq \vartheta & <\frac{\pi}{2} \\
-K_{B}(\vartheta) & : & \frac{5 \pi}{6} \leq \vartheta<\frac{7 \pi}{6} \\
-K_{A}(\vartheta) & : & \frac{7 \pi}{6} \leq \vartheta<\frac{9 \pi}{6}
\end{array}\right.
$$

Figure 5 shows the identified force functions. Sinusoids are applied to approximate the force functions, because the back-EMFs of the motor are nearly sinusoidal.

\subsection{Current Waveform Optimization}

Aim of the current waveform optimization is to obtain reference waveforms of the phase currents which generate smooth force. Inspection of the force equation(10) reveals that there are an infinite number of

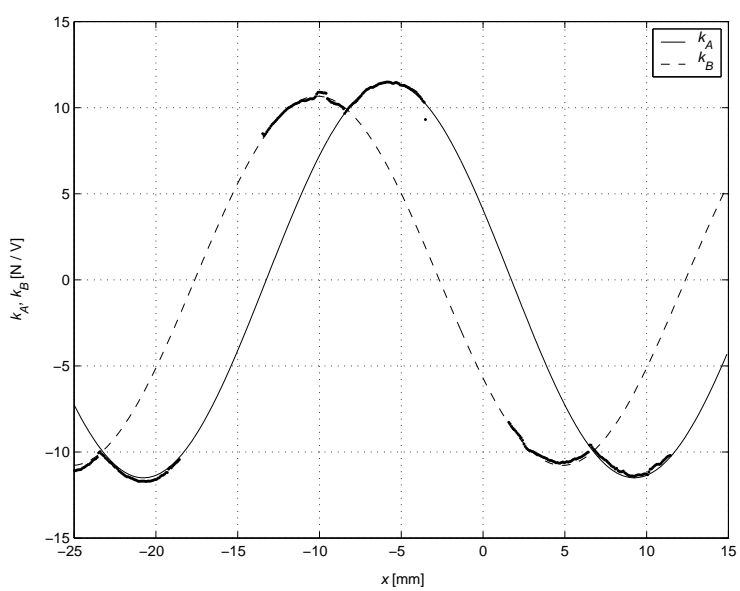

Figure 5: Identified force functions

waveforms that generates smooth force. Therefore a secondary condition has to be applied. Since one problem of epoxy-core PM LSM is overheating, the logical choice is to minimize the winding losses. The problem is formulated as constrained optimization. The constraint of the optimization is a position independent force

$$
F_{\text {thrust }}=K_{A}(\vartheta) u_{A}+K_{B}(\vartheta) u_{B}=K_{F} u \neq f(\vartheta)
$$

where $K_{F}$ is a freely eligible constant and $u$ is the force command. The winding losses can be written as

$$
P_{c u}(x)=\sum_{p} R_{p} i_{p}^{2}(x) ; \quad p \in\{A, B, C\}
$$

where $R_{p}$ is the resistance of phasewinding $p$. In assumption of symmetric winding resistances $R_{A}=$ $R_{B}=R_{C}$ and servo amplifier gains $K_{S_{A}}=K_{S_{B}}$ the functional to be minimized can be written

$$
f\left(u_{A}, u_{B}\right)=u_{A}^{2}+u_{B}^{2}+u_{A} u_{B} .
$$

If the resistances are unsymmetric and/or the gains are unequal and known, (14) has to be adapted to meet the requirements. In case of small unsymmetric resistances and gains, the minimization of (14) minimizes the winding losses approximately. After substituting (12) in (14) the optimized waveforms can be obtained by minimizing (14)

$$
\begin{aligned}
& u_{A}=\frac{\partial f\left(u_{A}, u_{B}\right)}{\partial u_{A}}=0 \\
& u_{B}=\frac{\partial f\left(u_{B}, u_{B}\right)}{\partial u_{B}}=0
\end{aligned}
$$


$u_{A}(u, \vartheta)=\frac{K_{A}(\vartheta)-\frac{1}{2} K_{B}(\vartheta)}{K_{A}^{2}(\vartheta)+K_{B}^{2}(\vartheta)-K_{A}(\vartheta) K_{B}(\vartheta)} K_{F} u$

$u_{B}(u, \vartheta)=\frac{K_{B}(\vartheta)-\frac{1}{2} K_{A}(\vartheta)}{K_{A}^{2}(\vartheta)+K_{B}^{2}(\vartheta)-K_{A}(\vartheta) K_{B}(\vartheta)} K_{F} u$

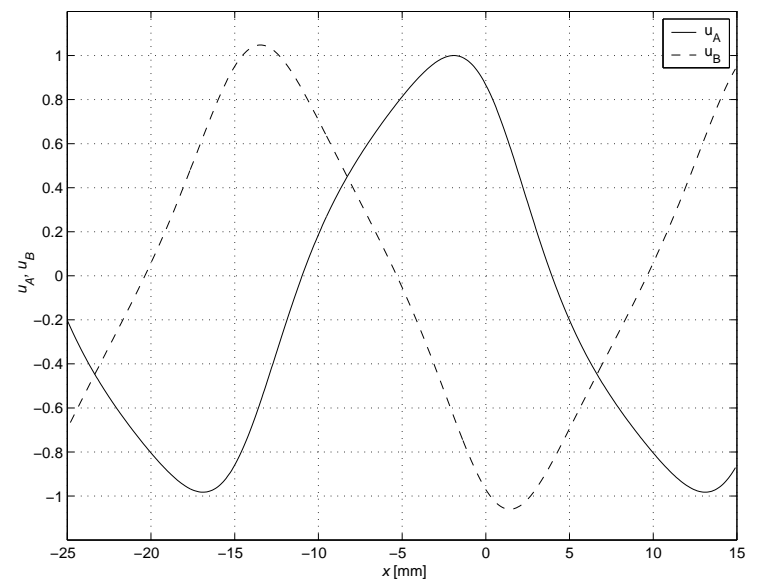

Figure 6: Optimized waveforms

In Figure 6 the optimized phase current waveforms are shown. Figure 7 compares the ripple of the force

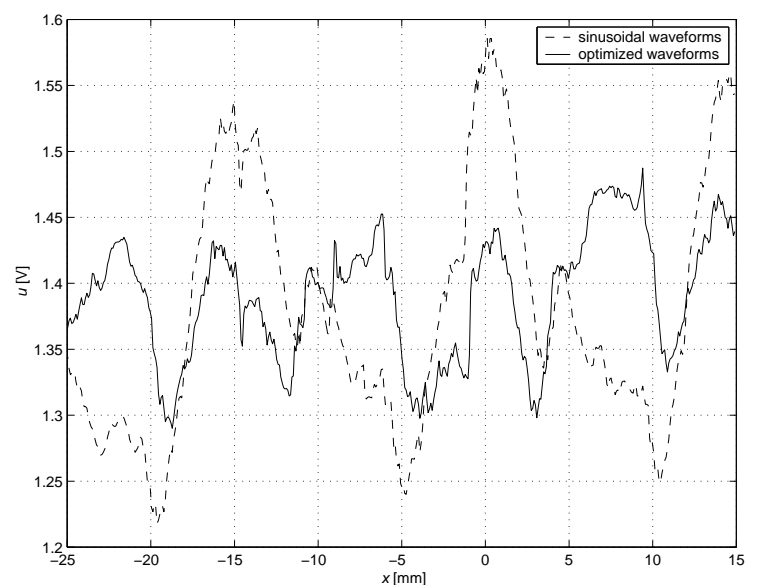

Figure 7: Force command signals with sinusoidal versus optimized waveforms

commands by use of different commutation functions. The dashed line shows the force command when a sinusoidal commutation plus offset compensation is employed (4). The solid line shows the force command when optimized waveforms are applied. The ripple of the force command is significantly reduced when the optimized waveforms are applied. The optimized waveforms maximize the motor efficiency by minimizing the ohmic winding losses.

\subsection{Fine tuning of waveforms}

After optimization of the waveforms the motor efficiency is maximized, but the force command still consists of some higher order ripple. The higher order ripple is caused by unmodeled harmonics of the force functions. In order to reduce some of the higher order ripple a fine tuning of the waveforms is performed. The main idea of the fine tuning algorithm is to measure the phase current control signals at constant load force. The fine tuning is performed with previously optimized waveforms in order to maximize motor efficiency. The still remaining higher order ripple of the force command modulates the optimized waveforms. The fine tuning algorithm approximates the shapes of the measured phase command signals with Fourier series. Figure 8 compares the measured phase command signals with the approximation. Figure $9 \mathrm{com}-$ pares the commutation functions of the second stage with the third stage.

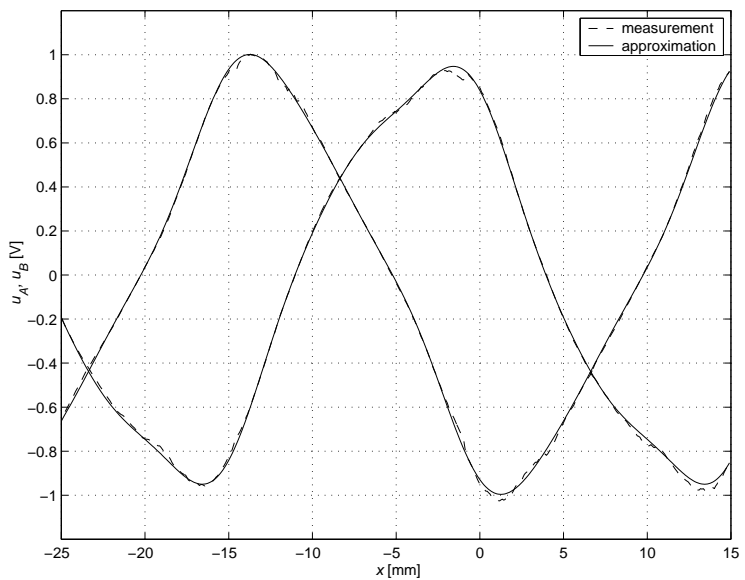

Figure 8: Fine Tuned Waveforms

$$
\begin{aligned}
u_{A}(u, \vartheta)= & u \frac{2}{3}\left(\sin (\vartheta)+a_{3} \sin \left(3 \vartheta+\alpha_{3}\right)+\right. \\
& \left.a_{5} \sin \left(5 \vartheta+\alpha_{5}\right)\right)+o_{A} \\
u_{B}(u, \vartheta)= & u \frac{2}{3}\left(\sin \left(\vartheta+\frac{2 \pi}{3}\right)+b_{3} \sin \left(3 \vartheta+\beta_{3}\right)+\right. \\
& \left.b_{5} \sin \left(5 \vartheta+\beta_{5}\right)\right)+o_{B}
\end{aligned}
$$




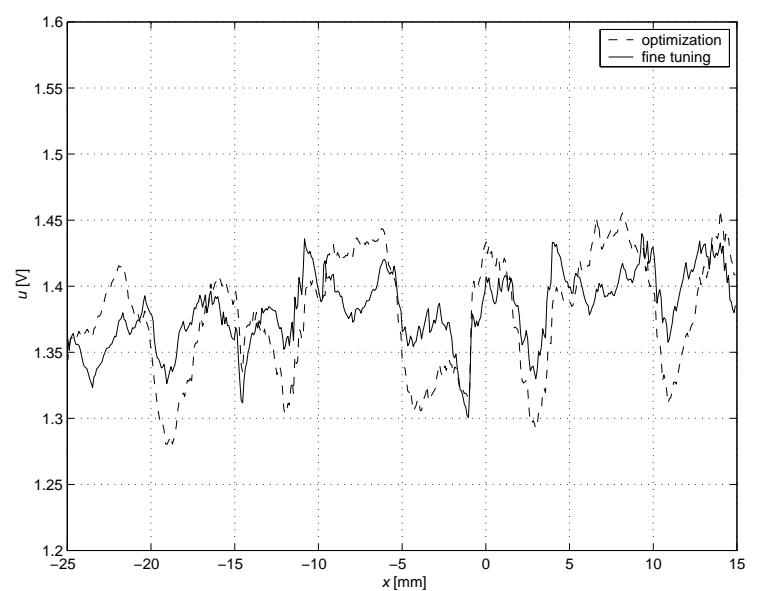

Figure 9: Force command signals with optimized versus fine tuned waveforms

Figure 10 compares the command signal spectrum of the optimization process. The upper left graph shows the spectrum before any optimization is applied. The upper right graph shows the spectrum after the offset compensation is applied. The fundamental is significantly reduced. In the lower left graph the spectrum of the force command of the optimized waveforms is shown. This stage reduces the $2^{\text {th }}$ order harmonics. The lower right graph shows the spectrum of the force command for fine tuned waveforms. This last stage reduces the $2^{\text {th }}$ and $4^{\text {th }}$ order harmonics. After the fine tuning of the waveforms no dominant harmonic exists in the force command.
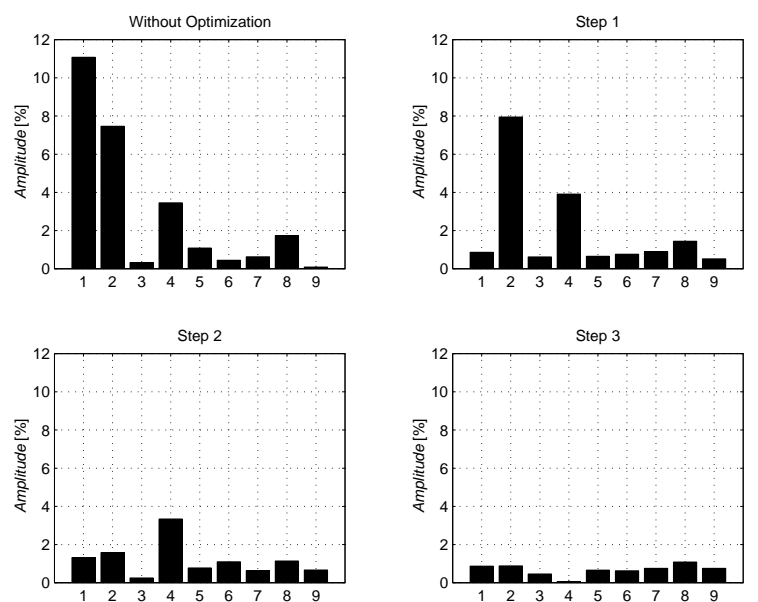

Figure 10: Spectrum of force command signals

\section{Controller Design}

Figure 11 shows the block diagram of the servo control system. In order to achieve a better tracking performance, a feedforward controller is applied. Feedback control without feedforward control always introduces a phase lag in the command response. Feedforward control sends an additional output, besides the feedback output, to drive the servo amplifier input to desired thrust force. The feedforward control compensates the effect of the carriage mass and the friction force. The friction force is modeled by a kinetic friction model and identified with experiments at different velocities. The mass of the carriage is identified with a dynamic least square algorithm. The stability of the system is determined by the feedback loop. The compensation of the force ripple is completely performed in the waveform generator with Fourier series approximation. Figure 12 compares the tracking error of a movement without ripple compensation with the movement with ripple compensation. In this measurement, the carriage moves from position $-25 \mathrm{~mm}$ to position $15 \mathrm{~mm}$ and back to position $-25 \mathrm{~mm}$ with $v_{\max }=200 \mathrm{~mm} / \mathrm{s}$. If the ripple compensation is applied, the tracking error is reduced significantly.

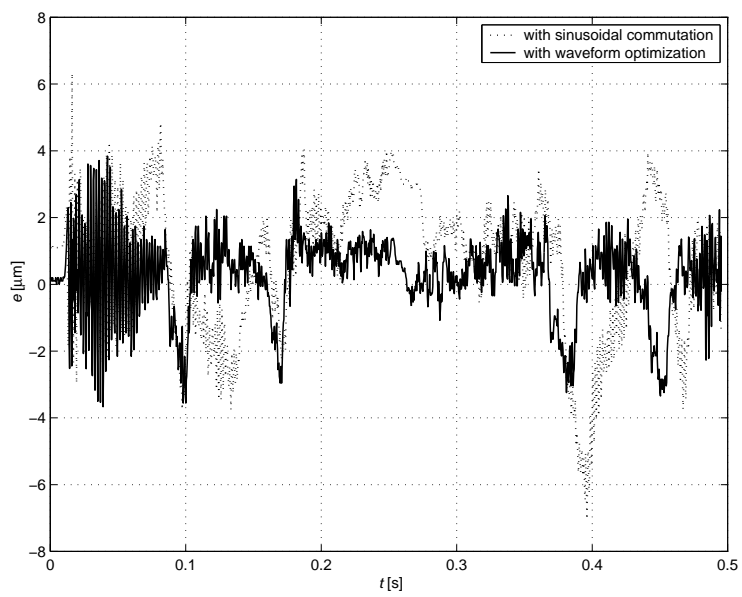

Figure 12: Tracking error

\section{CONCLUSION}

In this paper, a method for optimization of the current waveforms for PM LSMs is presented. The optimized waveforms generate smooth force and produce minimal copper losses which maximizes motor efficiency. The optimized current shapes are valid for any velocity and any desired thrust force. Since 


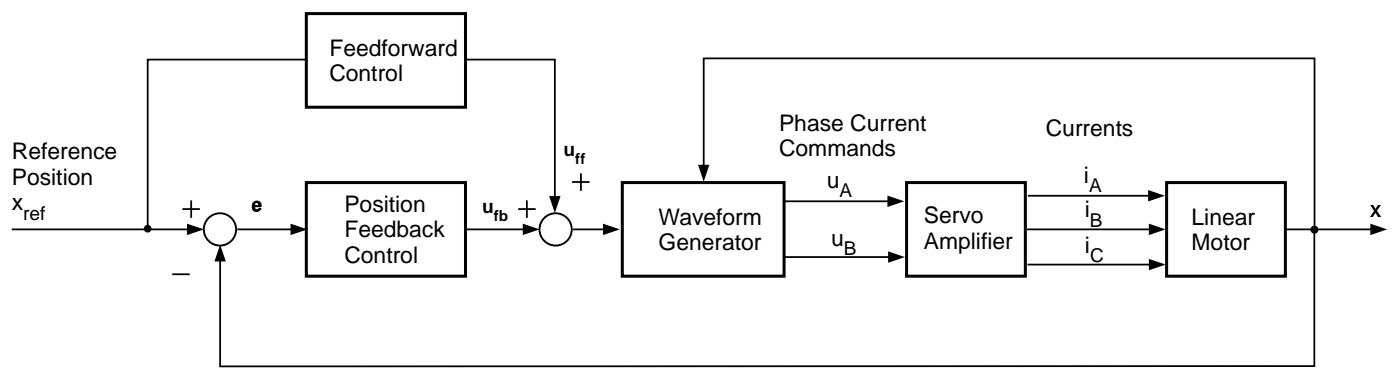

Figure 11: Controller design

the waveforms can be easily adapted to any shape of the back-EMF, motor design can be focused on increasing the mean force of the motor and reducing the cost of production. Experiments show that the tracking performance is significantly improved if the optimized waveforms are applied. The described optimization method is implemented successfully in the motion controllers of several machines for semiconductor production to improve the tracking performance.

\section{REFERENCES}

Anorad (1998). High Voltage Brushless D.C. Servo Amplifier, Hardware Maintenance Manual. Anorad Corporation.

Anorad (1999). LE Series Linear Motor Systems, Motor Integration Manual LEA, LEB, LEC \& LEM Linear Motors. Anorad Corporation.

Basak, A. (1996). Permanent-Magnet DC Linear Motors. Clarendon Press, Oxford.

Cassat, A., Corsi, N., Moser, R., and Wavre, N. (2003). Direct Linear Drives: Market and Performance Status. In Proceedings of the 4th International Symposium on Linear Drives for Industry Applications, pages 1-11, Birmingham, UK.

Gieras, J. and Zbigniew, J. (1999). Linear Synchronous Motors: Transportation and Automation Systems. CRC Press, Boca Raton.

Jahns, T. M. and Soong, W. L. (1996). Pulsating Torque Minimization Techniques for Permanent Magnet AC Motor Drives - A Review. IEEE Transactions on Industrial Electronics, 43(2):321-330.

Lee, T., Tan, K., Lim, S., and Dou, H. (2000). Iterative Learning of Permanent Magnet Linear Motor with Relay Automatic Tuning. Mechatronic, 10(1-2):169190.

Lin, F., Lin, C., and Hong, C. (2000). Robust Control of Linear Synchronous Motor Servodrive Using Disturbance Observer and Recurrent Neural Network Compensator. IEE Proceedings Electric Power Applications, 147(4):263-272.
Otten, G., de Vries, T., van Amerongen, J., Rankers, A., and Gaal, E. (1997). Linear Motor Motion Control Using a Learning Feedforward Controller. IEEE/ASME Transactions on Mechatronics, 2(3):179-187.

Röhrig, C. and Jochheim, A. (2001). Identification and Compensation of Force Ripple in Linear Permanent Magnet Motors. In Proceedings of the American Control Conference 2001, pages 2161-2166, Arlington, USA.

Röhrig, C. and Jochheim, A. (2002). Motion Control of Linear Synchronous Motors with Force Ripple Compensation using Current Shaping. In Proceedings of the 15th IFAC World Congress on Automatic Control, Barcelona, Spanien.

Schrijver, E. and van Dijk, J. (1999). $\mathrm{H}_{\infty}$ Design of Disturbance Compensators for Cogging Forces in a Linear Permanent Magnet Motor. Journal A, 40(4):36-41.

Seguritan, A. and Rotunno, M. (2002). Adaptive Torque Pulsation Compensation for a High-torque DC Brushless Permanent Magnet Motor. In Proceedings of the 15th IFAC World Congress on Automatic Control, Barcelona, Spanien.

Van den Braembussche, P., Swevers, J., and Van Brussel, H. (1998). Linear Motor Ripple Compensation Using Position-triggered Repetitive Control. In Proceedings of the IFAC Workshop on Motion Control, pages 353357, Grenoble, Frankreich.

Van den Braembussche, P., Swevers, J., Van Brussel, H., and Vanherck, P. (1996). Accurate Tracking Control of Linear Synchronous Motor Machine Tool Axes. Mechatronics, 6(5):507-521.

Xu, L. and Yao, B. (2000). Adaptive Robust Precision Motion Control of Linear Motors with Ripple Force Compensations: Theory and Experiments. In Proceedings of the IEEE Conference on Control Applications, pages 373-378, Anchorage, USA. 